\title{
Separation and Probiotic Effect of Fructose with Different Polymerization Degrees in Inulin
}

\author{
Mengcheng Ruan ${ }^{1}$,Tingting $\mathrm{Wu}^{1}$, Mengjun Cheng ${ }^{1}$, Jiale $\mathrm{Yu}^{1}$, Hualin Wang ${ }^{1}$, Zhiguo Liu $^{1 \mathrm{a}}$ \\ ${ }^{1}$ School of Biology and Pharmaceutical Engineering, Wuhan Polytechnic University, Wuhan 430023, China \\ * Correspondence:Zhiguo Liu: Tel.: +8615327270600,Email: zhiguo_1@126.com
}

\begin{abstract}
Inulin is a natural fructose polymer that can be used as a fat substitute in foods such as dairy products and bakery products and is often added to food products due to its effect on the regulation of intestinal flora (also known as the prebiotic effect). However, there are few studies exploring whether there are functional differences between fructose species with different degrees of polymerization (DP). Therefore, we focused on the separation of fructose species with different DP and their different effects on the balance of intestinal flora. First, the fractional precipitation method was used to separate short-chain inulin (DP:2-9), medium-chain inulin (DP:10-23), and long-chain inulin (DP:23 and above). Then, male C57BL/6 mice were randomly distributed into four groups and fed with a high-fat diet(HFD), a high-fat diet with added short-chain inulin(SCI), a high-fat diet with added medium-chain inulin(MCI), or a high-fat diet with added long-chain inulin(LCI) for two weeks. Finally, RT-PCR was used to detect the relative abundance of specific bacteria after this feeding course. The results showed that the abundance of common probiotics increased, and some harmful bacteria reduced after SCI, MCI, and HCI treatment. As the shortchain inulin has the strongest effect on improving the balance of intestinal flora, it may be a promising treatment option for patients with obesity, fatty liver, diabetes or other gastrointestinal issues.
\end{abstract}

\section{INTRODUCTION}

Along with the continued improvements in technology and productivity that society has experienced over the past decades has come an increased attention to personal health, with a particular interest in intestinal health. Intestinal flora can be divided into beneficial bacteria, harmful bacteria and neutral bacteria. The growth of these beneficial bacteria, commonly known as probiotics, can promote intestinal health, while high levels of harmful bacteria are linked with a variety of human diseases. The consumption of prebiotics, which serve as nutrient sources for these beneficial bacteria, not only promotes probiotic growth, but also inhibits excessive reproduction of harmful bacteria. A recent study has shown that inulin is the preferred raw material for bacteria and can therefore help maintain the balance of beneficial bacteria in the intestine. Specifically, inulin acts to promote an increase in the number of probiotics such that they become the dominant flora, while simultaneously inhibiting the proliferation of spoilage bacteria[1]. In addition, inulin has been suggested to regulate lipid metabolism, as well as prevent obesity and intestinal diseases.

While inulin is commercially available, these products are mixtures with different degrees of polymerization (DP) ranging from 2-60[2]. However, despite this clear heterogeneity, it remains unclear whether these inulin species differ in their ability to induce probiotic effects $[3,4]$. In this study, we began by separating the inulin raw materials, which entailed comparing different separation and purification methods and selecting a feasible scheme that was conducive to industrial large-scale production operations. Then, animal experiments were carried out by feeding mice diets containing inulin compounds with different DP to observe any differential regulation of the microbial community of intestinal flora. This worth, therefore, provides a critical foundation for the future development of inulin flour as a prebiotic food.

\section{MATERIALS AND METHODS}

\subsection{Separation and Identification of Inulin}

The method we used to separate Inulin was step precipitation method. The workflow was performed as previously described $[5,6]$. The corresponding quantity of the inulin raw material was weighed and dissolved in distilled water to prepare a saturated solution of inulin (provided by Wuhan Yingniulin Biotechnology Co., Ltd). Ethanol was then added to make a $10 \%$ ethanol solution, which was then centrifuged at $3010 \mathrm{rpm}$ for $10 \mathrm{~min}$ at $25^{\circ} \mathrm{C}$. The precipitate was collected and dried at $80^{\circ} \mathrm{C}$. Supernatant was collected for following process. This protocol was repeated to sequentially generate inulin solutions containing $20 \%, 30 \%, 40 \%, 50 \%, 60 \%, 70 \%$, 
$80 \%, 90 \%, 95 \%$, and $95 \%$ ethanol. The above dried sample was dissolved in distilled water, and the viscosity was determined by viscometry. The Mark-Houwink equation $\left([\eta]=\mathrm{KM}^{\alpha}\right)$ and $\mathrm{M}=162 \mathrm{DP}+180$ were used to calculate the average molecular weight of each sample[7,8].

\subsection{Animals and treatment}

Specific pathogen-free (SPF) Male C57BL/8 (12 weeks old, weighing 26-30g) were purchased from the Center for Disease Control of Hubei province (Wuhan, China) and were housed in a $12 \mathrm{~h}$ day/night cycle with a room temperature of $20 \pm 3^{\circ} \mathrm{C}[9]$. After one week of acclimatization, the mice were randomly distributed into the following four groups, with eight mice in each group: high-fat diet(HFD), high-fat diet with added short-chain inulin(SCI), high-fat diet with added medium-chain inulin(MCI), and high-fat diet with added long-chain inulin(LCI). The feed was produced and processed by Nantong Trophi Feed Technology Co.Ltd. Inulin was added at a mass ratio of $10 \%$ weight.

Mice were allowed to free-feed every day and given access to free drinking water. Every week, we collected their droppings, which were kept at $-80^{\circ} \mathrm{C}$ for downstream experiments[10].

\subsection{Quantification of intestinal bacteria by quantitative real-time PCR}

Fecal DNA was obtained using the TIANamp Stool DNA Kit (TIANGEN). Quantification was performed using 16S universal or bacterial specific rRNA gene primers and quantitative RT-PCR [11-13]. Differences $(\triangle \mathrm{CT})$ between $16 \mathrm{~S} \mathrm{CT}$ values and specific bacterial groups were used to obtain normalized levels $(2-\triangle \mathrm{CT})$. The relative abundance of each bacterial group was obtained after normalization with the control groups. Primer sets used for qPCR are listed in Table 1.

Table 1. Primer sequences

\begin{tabular}{|c|c|c|}
\hline Targeted bacterial & $\begin{array}{l}\text { Code } \\
\text { name }\end{array}$ & Sequence $\left(5^{\prime}->3^{\prime}\right)$ \\
\hline $\begin{array}{c}\text { Bacteroides- } \\
\text { Prevotella- } \\
\text { Porphyromonas }\end{array}$ & BPP & $\begin{array}{l}\text { F: GGTGTCGGCTTAAGTGCCAT } \\
\text { R:CGGAYGTAAGGGCCGTGC }\end{array}$ \\
\hline Lactobacillus spp. & LAC & $\begin{array}{c}\text { F:AGAGGTAGTAACTGGCCTTT } \\
\text { A R:GCGGAAACCTCCCAACA }\end{array}$ \\
\hline Ruminococcaceae & RUM & $\begin{array}{c}\text { F:GAGTGAAGTAGAGGTAAGC } \\
\text { GGAATTC } \\
\text { R:GCCGTACTCCCCAGGTGG }\end{array}$ \\
\hline Enterobacteriaceae & ENB & $\begin{array}{c}\text { F:ATGGCTGTCGTCAGCTCGT } \\
\text { R:CCTACTTCTTTTGCAACCCA } \\
\text { CTC }\end{array}$ \\
\hline Clostridium leptum & CLL & $\begin{array}{c}\text { F:GCACAAGCAGTGGAGT } \\
\text { R:CTTCCTCCGTTTTGTCAA }\end{array}$ \\
\hline $\begin{array}{l}\text { Clostridium cluster } \\
\text { XIVab }\end{array}$ & $\mathrm{CCX}$ & $\begin{array}{c}\text { F:GCAGTGGGGAATATTGCA } \\
\text { R:CTTTGAGTTTCATTCTT }\end{array}$ \\
\hline GenusPrevotella & GEN & $\begin{array}{c}\text { F:CACRGTAAACGATGGATGCC } \\
\text { R:GGTCGGGTTGCAGACC }\end{array}$ \\
\hline $\begin{array}{l}\text { Bifidobacterium } \\
\text { spp }\end{array}$ & BIF & $\begin{array}{c}\text { F:CGCGTCCGGTGTGAAAG } \\
\text { RCTTCCCGATATCTACACATTC } \\
\text { CA }\end{array}$ \\
\hline Enterococcus spp & ENS & $\begin{array}{c}\text { F:CCCTTATTGTTAGTTGCCATC } \\
\text { ATT }\end{array}$ \\
\hline
\end{tabular}

\begin{tabular}{|c|c|c|}
\hline & & $\begin{array}{c}\text { R:ACTCGTTGTACTTCCCATTG } \\
\text { T }\end{array}$ \\
\hline Firmicutes & FIR & $\begin{array}{c}\text { FGGAGYATGTGGTTTAATTCG } \\
\text { AAGCA } \\
\text { RAGCTGACGACAACCATGCAC }\end{array}$ \\
\hline Total bacteria & $16 \mathrm{~s}$ & $\begin{array}{l}\text { F:GTGSTGCAYGGYYGTCGTCA } \\
\text { R:ACGTCRTCCMCNCCTTCCTC }\end{array}$ \\
\hline
\end{tabular}

\subsection{Statistical analysis}

Data are presented as mean $\pm \mathrm{SD}$. A one-way ANOVA and Tukey's post hoc analysis were conducted to establish significant differences. All analyses were performed with Graphpad Prism 7.0 (GraphPad, La Jolla CA,USA). Differences were considered statistically significant at threshold of $\mathrm{p}<0.05$.

\section{RESULTS}

\subsection{Separation of inulin}

Inulin raw materials was dissolved in distilled water, and the viscosity was determined by viscometry. Step precipitation method was adopted to separate the inulin with DP range from 1.1 to 42 DP. Details were showed in table 2 .

Table 2. Results of Inulin Purification Scheme

\begin{tabular}{|c|c|c|c|c|c|}
\hline $\begin{array}{l}\text { Ethanol } \\
\text { concentr } \\
\text { ation(\%) }\end{array}$ & $\begin{array}{l}\text { quality } \\
(\mathrm{g})\end{array}$ & $\begin{array}{l}\text { Inherent } \\
\text { viscosity } \\
\left(\mathrm{ml} \cdot \mathrm{g}^{-1}\right)\end{array}$ & DP & Group & $\begin{array}{c}\text { Content } \\
(\%)\end{array}$ \\
\hline 10 & 12.75 & 7.31 & 42 & \multirow{3}{*}{$\begin{array}{l}\text { Long- } \\
\text { chain }\end{array}$} & 12.75 \\
\hline 20 & 6.71 & 6.11 & 33 & & 6.71 \\
\hline 30 & 2.52 & 5.56 & 23 & & 2.52 \\
\hline 40 & 7.66 & 4.91 & 20 & \multirow{3}{*}{$\begin{array}{l}\text { Medium } \\
\text {-chain }\end{array}$} & 7.66 \\
\hline 50 & 15.89 & 4.20 & 17 & & 15.89 \\
\hline 60 & 11.32 & 3.60 & 13 & & 11.32 \\
\hline 70 & 10.35 & 3.20 & 10 & \multirow{5}{*}{$\begin{array}{l}\text { Short- } \\
\text { chain }\end{array}$} & 10.35 \\
\hline 80 & 9.68 & 2.82 & 8 & & 9.68 \\
\hline 90 & 5.11 & 2.45 & 5 & & 5.11 \\
\hline 95 & 4.89 & 2.11 & 2.5 & & 4.89 \\
\hline $95+$ & 2.95 & 1.71 & 1.1 & & 2.95 \\
\hline
\end{tabular}

\subsection{Short-chain inulin significantly promoted the growth of probiotics}

To understand the effect of inulin treatment on probiotic composition, the common probiotics Lactobacillus(LAC), Bifidobacterium(BIF), and Ruminococcaceae(RUM)were used as proxies for microbiome diversity. The SCI treatment group showed signifciantly increased levels of all three species when compare to the HFD group. Conversely, no significant changes in bacterial community composition were identified in the MCI and LCI groups. These results suggest that short-chain inulin can promote growth of LAC, BIF, and RUM, while medium- and long-chain inulin cannot induce this response. 
Prevotella(GEN) is a commensal bacterium that is widely distributed in humans as neutral bacterium. Results showed that there were not any changes between HFD and SCI, MCI, while LCI can significantly reduce the aboundance of it.

Thus, the addition of short-chain inulin to feed is sufficient to increase probiotic diversity in the mouse intestinal microbiome.
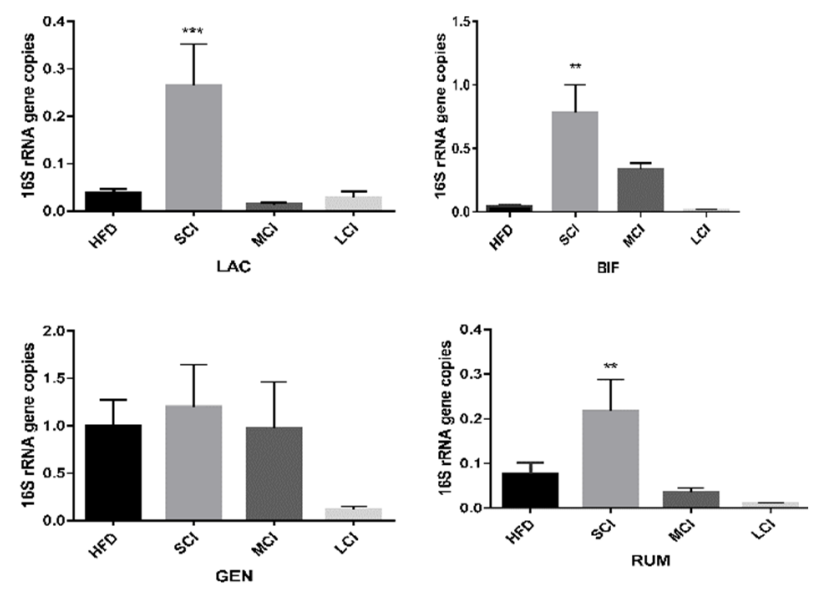

Figure 1. Effects of different DP inulin on intestinal probiotic levels. Data are expressed as mean $\pm \mathrm{SD}$ ( $\mathrm{n}=8$ per group). ${ }^{*} \mathrm{p}<0.05,{ }^{* *} \mathrm{p}<0.001,{ }^{* * *} \mathrm{p}<0.001$ vs HFD group.

\subsection{Short-chain inulin significantly inhibited the growth of harmful bacteria}

Harmful bacteria, including Bacteroides (BPP), Enterobacteriacaea (ENB), Enterococcus (ENS), Clostridiumleptum (CLL), Firmicute (FIR) and Clostridium cluster XIVab (CCX), were found at high levels in the HFD group. However, their presence was significantly decreased in the SCI group, as well as (to a lesser degree) in the MCI and LCI groups. These results suggest that inulin is capable of inhibiting growth of common harmful bacteria regardless of DP, but that short-chain inulin does so most effectively.
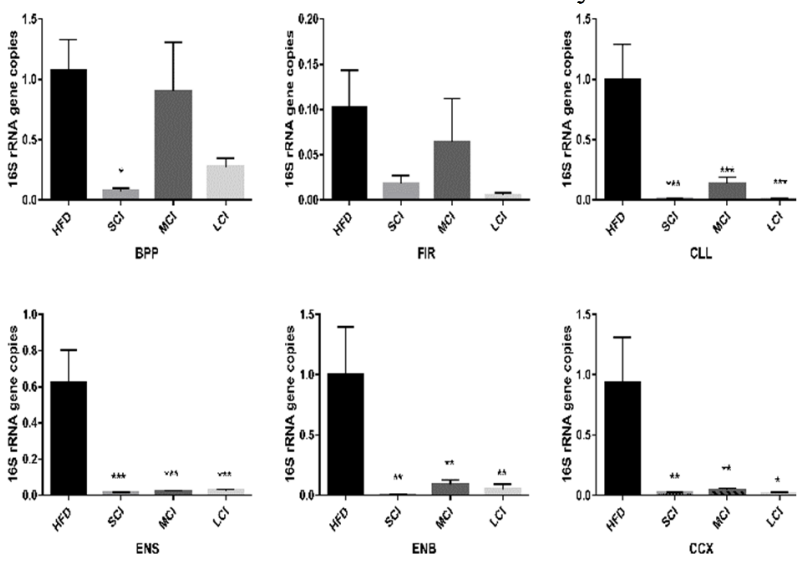

Figure 2. Effect of inulin with different degrees of polymerization on the prevalence of harmful intestinal bacteria Data are expressed as mean $\pm \mathrm{SD}\left(\mathrm{n}=8\right.$ per group). ${ }^{*} \mathrm{p}<0.05$, $* * \mathrm{p}<0.001, * * * \mathrm{p}<0.001$ vs HFD group.

\section{DISCUSSION}

Inulin has attracted the attention of many researchers in recent years. There have been many studies on the separation method of inulin, most of which employ organic solvent step precipitation method or highperformance gel permeation chromatography. The former approach leverages the different solubility properties of different polysaccharides in alcohols and ketones. Therefore, alcohols and ketones can be added in specific proportions to precipitate the desired polysaccharides, as employed to isolate inulin in this research. The latter approach was applied by Ronkart[14], who hydrolyzed inulin by inulinase and then performed High Performance Size Exclusion Chromatography (HPSEC) to isolate inulin with a degree of polymerization of 2-4.Yang et al [15] used different gel fillers to extract and separate low-polymerization forms of inulin and ESI-MS confirmed successful isolation of products with DPs between two and nine.

Comparing these approaches, HPSEC has the advantages of higher speed, efficiency, and precision, but the disadvantage is that each separation, the amount of product we can get is very small.t. The ethanol precipitation method is highly accurate and relatively simple to perform. Furthermore, this method can be scaled to generate large amounts of product for a low price, making this approachmore suitable for large-scale production such as factories. However, this technique is not sensitive enough to discriminate single-degree differences between polymer species. The experimental operation accounting for a large proportion, etc, which need to be improved in the future.

A growing body of research shows that the intestinal flora of animals and humans has clear effects on host health. However, the high-fat, high-protein diet of many modern people has led not only to a decrease in the overall intestinal flora population, but also to reduced species diversity. This disordered intestinal flora can have far-reaching and serious effects on human health. Previous studies have found that the consumption of inulin as a dietary fiber can provide food for the hungry gut microflora and stimulate the growth of intestinal probiotics in cases where the number of flora has been reduced by a high-fat diet. Additional studies have shown that a diet supplemented with inulin can stimulate the growth of probiotics and inhibit the colonization of harmful bacteria, thereby reducing intestinal inflammation [16]. However, previous research has failed to explore the role that inulin DP mayplay in promoting or inhibiting these effects.

Our study showed that, as compared to the HFD group, the SCI group exhibited increased levels of probiotics Lactobacillus, Bifidobacterium, Ruminococcaceae, and Prevotella. As well as decreased levels of harmful bacteria (Bacteroides, Enterobacteriaceae, Enterococcus, Clostridium, and Firmicute). These findings are consistent with those of $\mathrm{Li}$ et al. [17], who showed that inulin treatment led to a 510 times increase in Bifidobacterium, as well as impaired proliferation of potentially harmful bacteria[18]. 
Although our results showed that SCI, MCI, and LCI treatment groups all exhibited significantly decreased levels of Enterobacteriaceae and Enterococcus, the immense diversity across even a single bacterial genus means that further research is necessary to fully elucidate the physiological significance of these changes [19].

\section{CONCLUSION}

In conclusion,consumption of a high-fat diet supplemented with inulin induced changes to the makeup of murine intestinal flora, although these changes were observed after treatment with short-chain, medium-chain, and high-chaininulin, short-chain inulin was the most effective at both promoting probiotic growth and inhibiting the growth of harmful bacteria.

\section{Acknowledgements :}

This study was supported by Wuhan science and technology project (Grant No. 2018020402011230), and the central government guides local science and technology development projects (Grant No. 2019ZYYD 032).

\section{REFERENCE}

1. L.N.Yang, K.W.Wu, L.J.Zhu. J. Science and Technology of Food Industry, 21,336-340 (2007)

2. D.Luo. J. Food Science,38,67-73 (2017)

3. Y.Zhang. D.Qingdao University of Science \& Technology (2016)

4. J.Y.Liu. D.Dalian Polytechnic University (2013)

5. X.Lee, J.Shi. J. Food research and development, 34,51-55 (2013)

6. Z.X.Chen, S.Zheng. Journal of Shangdong of College of textle technology. 7,44-46 (1992)

7. H.Q.Yang. Chemical Education, 01,61 (1985)

8. Y.T.Sun, H.H.Xu. China Journal of Chinese Materia Medica.8,1-9(2019)

9. D.J.Streeter, R.F.Boyer. J Polymer Sci.14,5-14(1954)

10. H.Robert,S.Anthony,W.Richard,H.Pravin,P. Nikolai. The Journal of allergy and clinical immunology. 144,25(2019)

11. S.K.Guo,W.M.Bao. Chinese Journal of Microecology. 22,24-28(2010)

12. P.Bai, Y.M.Lu. Chinese Journal of Gastroenterology and Hepatology.17,566-571 (2008)

13. T.Rintlia, A.Kassinen, E.Malinen. Journal of applied microbiology.6,1166-1177(2004)

14. S.N.Ronkart, C.S.Blecker, H.Fourmanoir, C.Fougnies, C.Deroanne. Analytica Chimica Acta.604,81-87(2007)

15. Z.Yang, Y.Yi,C. Gao, D.Hou, J.Hu, M.Zhao. Journal of Separation Science.33, 120-125(2000)
16. F.Guarner. Journal of Nutrition. 137,2568S-2571S (2007)

17. Z.Z.Li, Y.H.Liu. Chinese Journal of Microecology. 27,1365-1369 (2015)

18. Z.L.Zhou, L.K.Liao, R,Y. Wu. Progress in Modern Biomedicine.17,4201-4206,4258(2017)

19. C.Zhang, L.Zhao. Genome Medicine. 8 ,41(2016) 